\title{
Incidence of acquired lacrimal drainage system obstruction in epiphoric patients in Thailand
}

\author{
Suppapong Tirakunwichcha ${ }^{\mathrm{a}}$, Ehwika Rengwanidchakul ${ }^{\mathrm{b}}$, Somkiat Asawaphureekorn ${ }^{\mathrm{b}}$, Supaporn Tengtrisorn ${ }^{\mathrm{c}}$, \\ Khanchai Juangphanich ${ }^{\mathrm{d}}$, Pitipong Suramethakul ${ }^{\mathrm{e}}$, Kanokrat Pornpanich ${ }^{\mathrm{f}}$ \\ ${ }^{a}$ Department of Ophthalmology, Faculty of Medicine, Chulalongkorn University, Bangkok 10330; \\ ${ }^{b}$ Department of Ophthalmology, Srinagarind Hospital, Faculty of Medicine, Khon Kaen University, \\ Khon Kaen 40002; 'Department of Ophthalmology, Songklanagarind Hospital, Faculty of Medicine, \\ Prince of Songkla University, Songkhla 90110; ${ }^{d}$ Department of Ophthalmology, Phramongkutklao \\ Hospital, Bangkok 10400; ${ }^{e}$ Department of Ophthalmology, Mettapracharak Hospital, Nakornphathom

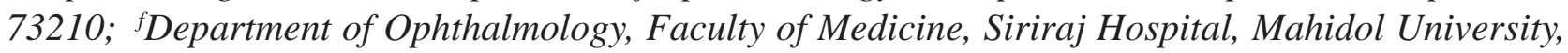 \\ Bangkok 10700, Thailand
}

\begin{abstract}
Background: There were few reports regarding the incidence of the acquired nasolacrimal duct obstruction, and the incidence in different geography or countries might be alike. Since the nasolacrimal drainage system obstruction is a common problem in Thailand, knowledge of the incidence may disclose the burden of the disease that has never been reported.

Objectives: Evaluate the incidence of acquired lacrimal drainage system obstruction (ALDO) in Thailand.

Methods: This study was conducted in multi-centers. It was based on prospective and descriptive design. All patients with epiphoric symptoms visiting the outpatient departments of five tertiary eyecare centers were recruited between 2004 and 2007. Features of epiphoric symptoms and medical history were recorded, and complete ophthalmic examinations were performed.

Results: Epiphoric patients were enrolled from all medical centers with 245 eligible patients, female:male ratio was 4.2:1. Ninety-nine patients ( $40 \%$ ) had duration of epiphoric symptoms between one-five years. ALDO was found in 111 patients $(45 \%)$ with female preponderance $(6.9: 1)$. Seventy-one patients $(64 \%)$ had pre-sac obstruction and 40 patients $(36 \%)$ had post-sac obstruction. Among other causes of epiphora, dry eye was the most common. Conclusion: The most common cause of epiphora was ALDO. The four-year incidence rate of ALDO among epiphoric patients was $45 \%$.
\end{abstract}

Keywords: Dacryocystitis, epidemiology, epiphora, incidence, lacrimal duct obstruction

Lacrimal drainage system obstruction is a disease where the obliteration occurs at any parts of the lacrimal excretory pathway: the offset at puncta, upper/lower canaliculi, common canaliculus, and nasolacrimal duct through the opening at the inferior meatus. Patients present with different degrees of symptoms e.g., epiphora, ocular discharge, conjunctivitis, dacryocystitis, mass at the lacrimal sac,

Correspondence to: Dr. Suppapong Tirakunwichcha, Department of Ophthalmology, Faculty of Medicine, Chulalongkorn University, 1873 Rama IV Road, Patumwan, Bangkok 10330, Thailand. E-mail: suppapong.t@chula.ac.th localized cellulitis, or abscess formation, even though orbital cellulitis.

Regarding the onset of the obstruction, focusing on nasolacrimal duct, it can be classified into congenital and acquired groups. In the published literatures, only a few articles [1-3] reported the incidence of congenital nasolacrimal duct obstruction and very few articles $[4,5]$ reported the incidence of acquired lacrimal drainage system obstruction (ALDO). The objective of this study is to establish the incidence of ALDO in epiphoric patients that presented to the tertiary eyecare hospitals in Thailand. 


\section{Materials and methods}

Patients with epiphoric symptoms, older than 18 years old, who presented at the out-patient clinics of five tertiary eyecare hospitals in Thailand; three medical centers in Bangkok (King Chulalongkorn Memorial Hospital, Phramongkutklao Hospital, and Siriraj Hospital, Bangkok), one medical center in the Northeast (Srinagarind Hospital, Khon Kaen) and one medical center in the south (Songkhlanagarind Hospital, Songkhla) between 2004 and 2007 were recruited into the study. Patients with any history of previous trauma or surgery around the eye that might injure the lacrimal drainage apparatus were excluded. The epiphoric symptoms and previous medical history were recorded. Complete ophthalmic examinations using slit-lamp biomicroscopy were performed to rule out other diseases or conditions that induced epiphora rather than lacrimal drainage obstruction. Snap back test was checked to evaluate the eyelid laxity and tear film break-up time were also assessed. Lacrimal drainage system obstruction was confirmed by fluid irrigation with normal saline in a three-mL syringe attached to the 25 -gauge irrigating needle. This study was approved by the Ethics Committee of all participating institutions and informed consents were obtained from all participants.

\section{Results}

Three hundred and fifteen patients with epiphora were enrolled into the study from all five medical centers. Seventy patients were not eligible because of incomplete data. Two hundred forty five patients were used for data analysis.

Most patients with epiphora were female (198 out of 245 patients, $80.8 \%$ ) (Table 1). Compared to male, female had epiphora about 4.2 times. The mean age of epiphora was 58 (SD: 14) years old. Ninety-nine patients $(40.4 \%)$ had epiphoric symptoms between 15 years, $30.6 \%$ had less than one year and $29.0 \%$ had more than five years. One hundred and eighteen patients $(48.2 \%)$ had symptoms on both sides. Sixty- five patients $(26.5 \%)$ and 62 patients $(25.3 \%)$ had symptoms only on the right side or the left side, respectively. Regarding the patients with ALDO (111 out of 245 patients), female was preponderant ( 97 out of 111 patients, $87.4 \%$ ) and was about sevenfold more than male. The mean age was 61 years old (SD: 15). The duration of symptoms was mainly between 1-5 years $(52.3 \%)$.

Most epiphoric patients presented without any discharge (186 out of 245 patients, $75.9 \%$ ) (Table 2 ), compared to around half of the patients (58 out of 111 patients, 52.3\%) with ALDO. Thirty-four patients (13.9\%) experienced epiphora with mucopurulent secretions, and 25 patients $(10.2 \%)$ had a preceding mass or abscess formation over the lacrimal sac area. Snap back test was positive in 21 cases $(8.6 \%)$ : 13 cases with ALDO and 8 cases with non-ALDO.

Table 1. Number, mean age, laterality and duration of patients with epiphora and patients with acquired lacrimal drainage system obstruction (ALDO).

\begin{tabular}{lll}
\hline & Patients with epiphora & Patients with ALDO \\
\hline $\begin{array}{ll}\text { Patients, number (\%) } \\
\quad \text { Male }\end{array}$ & $47(19.2 \%)$ & $14(12.6 \%)$ \\
$\quad$ Female & $198(80.8 \%)$ & $97(87.4 \%)$ \\
$\quad$ All & $245(100.0 \%)$ & $111(100.0 \%)$ \\
Mean age, year (SD) & $58(14)$ & $61(15)$ \\
Laterality, \% & & \\
$\quad$ Right & 26.5 & 31.5 \\
$\quad$ Left & 25.3 & 33.3 \\
$\quad$ Bilateral & 48.2 & 35.2 \\
Duration, number (\%) & \\
$\quad<1$ year & $75(30.6 \%)$ & $32(28.8 \%)$ \\
1-5 years & $99(40.4 \%)$ & $58(52.3 \%)$ \\
$\quad$ 5 years & $71(29.0 \%)$ & $21(18.9 \%)$ \\
\end{tabular}


Table 2. Presenting symptoms in patients with epiphora and patients with acquired lacrimal drainage system obstruction (ALDO).

\begin{tabular}{lll}
\hline & $\begin{array}{l}\text { Patients with epiphora } \\
\text { number (\%) }\end{array}$ & $\begin{array}{l}\text { Patients with ALDO } \\
\text { number (\%) }\end{array}$ \\
\hline Epiphora without discharge & $186(75.9 \%)$ & $58(52.3 \%)$ \\
Epiphora with mucopurulent discharge & $34(13.9 \%)$ & $28(25.2 \%)$ \\
Mass over lacrimal sac area & $22(9.0 \%)$ & $22(19.8 \%)$ \\
Abscess over lacrimal sac area & $3(1.2 \%)$ & $3(2.7 \%)$ \\
\hline
\end{tabular}

Out of the 111 patients $(45.3 \%)$ who had obstructions of the lacrimal drainage system, 14 patients (12.6\%) had reflux back around the shaft of the irrigating needle, 97 patients $(87.4 \%)$ had reflux through the opposite punctum (57 patients, 51.4\%) without discharge and high velocity reflux, and 40 patients $(36.0 \%)$ with mucoid or mucopurulent discharge).

\section{Discussion}

ALDO is a common disease in the developing countries and frequently found in the low socioeconomic population. Patients are usually presented with tearing all day or periodically. Some patients may have bulging masses or abscesses over the lacrimal sac areas. There are few reports about the incidence of ALDO. In 1964, Dalgleish [4] reported $22 \%$ incidence rate of ALDO by performing a routine irrigation of the lacrimal drainage system in both eyes prior to intraocular surgery in 3,487 patients. Recently, Woog [5] made retrospective review of the Rochester Epidemiology Project, which was done in Olmsted County, Minnesota between 1976 and 2000, and reported the incidence of symptomatic ALDO of 30.47 per 100,000 population.

In our multi-center tertiary eyecare hospitals study, all patients who complained of epiphora were enrolled and complete eye examinations were performed. All etiologies of epiphora were carefully determined. During four years of the study, almost half of the epiphoric patients (45.3\%) had ALDO, which $64.0 \%$ was at Bangkok, while $36.0 \%$ was in the other centers. This might imply the referring to the capital city rather than the authentic incidence in different geography. The very high ratio of female-to-male (6.9:1) in patients with ALDO may be a result of high ratio of female in the entire epiphora patients $(4.2: 1)$. This preponderance for female in our study is in concordance with a previous report by Woog [5]. The average age and the duration of symptoms (1-5 years) of patients with ALDO and all patients with epiphora were quite similar. The patients with epiphora had symptoms bilaterally in twofold than unilaterally, while patients with ALDO had nearly the same percentage in each group.

Most patients with epiphora had epiphora without discharge $(75.9 \%)$, and more than half $(52.3 \%)$ of patients with ALDO presented as well. About $82 \%$ of the epiphoric patients who presented with mucopurulent discharge (28 out of 34 patients) had ALDO. Not surprisingly, all epiphoric patients who presented with mass or abscess over the lacrimal sac area had ALDO. The mean age of patients with ALDO was 61 years old. This was similar to that reported by Woog [5], but his most common site of the obstruction located at the nasolacrimal duct $(67.6 \%)$. This was different from our most common site of obstruction, common canaliculus (51.4\%). For patients without drainage system obstructions (134 patients, $54.7 \%$ ), the most common cause of epiphora was dry eye $(58.2 \%)$. Other causes were corneal surface irregularities, concretions of the upper eyelids, eyelid laxity, trichiasis/distichiasis, conjunctival congestion, and facial palsies, etc.

There are several factors affecting the incidence rate of ALDO in this study. Since the study was conducted in the tertiary eyecare hospitals, where the patients with ALDO were already screened and referred from primary or secondary eyecare hospitals. Other factors might be the selection bias at the outpatient clinics by the screening nurses, and the time limitation for the oculoplastic surgeon in caring for all patients who complained of epiphora. To our knowledge, our study is the first study that reported the incidence of ALDO in patients with epiphora in Thailand. The study covered patients not only from the central area but also from the northeast and the southern part of Thailand. However, since the study 
was conducted in tertiary eyecare hospitals, the reported incidence would be higher than it would be. A community-based study conducted with larger sample size covering all parts of the country should be pursued to determine the true incidence of ALDO in patients with epiphora.

\section{Conclusion}

The most common cause of epiphora was ALDO. It occurred at the mean age of 61 years old. Female were predominant about seven times than male. Both patients with epiphora and patients with ALDO had the same duration of one-five years. The most common site of obstruction was pre-sac (64\%). The four-year incidence rate of ALDO among epiphoric patients was $45 \%$.

\section{Acknowledgement}

This study was supported by Ratchadapiseksompotch Fund. No author has a financial interest, commercial associations or conflict of interest in any aspect of this report.
All authors had made significant contributions in this work.

\section{References}

1. Noda S, Hayasaka S, Setogawa T. Congenital nasolacrimal duct obstruction in Japanese infants: its incidence and treatment with massage. J Pediatr Ophthalmol Strabismus. 1991;28:20-2.

2. Hick JF, Block D, Ilstrup DM. A controlled study of silver nitrate prophylaxis and the incidence of nasolacrimal duct obstruction. J Pediatr Ophthalmol Strabismus. 1985; 22:92-3.

3. Macewen CJ, Young JDH. Epiphora during first year of life. Eye. 1991; 5:596-600.

4. Dalgleish R. Incidence of idiopathic acquired obstructions in the lacrimal drainage apparatus. $\mathrm{Br} \mathrm{J}$ Ophthalmol. 1964; 48:373-6.

5. Woog JJ. The incidence of symptomatic acquired lacrimal outflow obstruction among residents of Olmsted County, Minnesota, 1976-2000 (an American Ophthalmological Society Thesis). Trans Am Ophthalmol Soc. 2007; 105:649-66. 